\title{
Affective Bias and Response Modulation Following Tyrosine Depletion in Healthy Adults
}

\author{
Suzanne Vrshek-Schallhorn', Dustin Wahlstrom ${ }^{1,2}$, Kelly Benolkin', Tonya White ${ }^{2,3}$ and Monica Luciana*,1,2 \\ 'Department of Psychology, University of Minnesota, Minneapolis, MN, USA; ${ }^{2}$ Center for Neurobehavioral Development, University of Minnesota, \\ Minneapolis, MN, USA; ${ }^{3}$ Department of Psychiatry, University of Minnesota, Minneapolis, MN, USA
}

\begin{abstract}
Acute phenylalanine/tyrosine depletion (ATPD) has been used to transiently lower central nervous system dopamine activity in animals and humans. Findings suggest that ATPD may impact dopamine transmission in limbic and striatal regions. Impact on cognitive functions has varied across studies, although several recent reports suggest that affective processing in the context of a go/no-go response control task may be impaired during ATPD. In this study, response control under affective vs nonaffective conditions was examined in healthy individuals who underwent either ATPD or a balanced condition in a between-subjects design. Effects of ATPD were validated through its effects on serum prolactin secretion. ATPD resulted in significantly increased prolactin levels relative to the balanced mixture. Although there were no differences in self-reported mood between the groups, individuals in the ATPD condition demonstrated diminished sensitivity to positively valenced words and seemingly enhanced sensitivity to negatively valenced words in an affective go/no-go task. They also showed difficulties in modulating ongoing behavior in a nonaffective go/no-go task when responses had to be intermittently inhibited then immediately restarted. Basic motor functions were not impacted. Findings are discussed in relation to dopamine's role in switching signals within neural networks that are important for response modulation and affective control. Neuropsychopharmacology (2006) 3 I, 2523-2536. doi:I 0. I038/sj.npp. I 30I I 72; published online 26 July 2006
\end{abstract}

Keywords: tyrosine depletion; dopamine; affect; go/no-go; cognitive control; anhedonia

\section{INTRODUCTION}

Pharmacological probes of neurochemical systems in humans using agonist and antagonist compounds have been employed to examine their roles in modulating various information processing functions. Recently, acute tyrosine/ phenylalanine depletion (ATPD) has been proposed as an effective means of lowering central nervous system dopamine (DA) activity by reducing precursor availability. ATPD involves administration of an amino-acid beverage that is deficient in DA's amino-acid precursors, tyrosine, and phenylalanine. Several hours after ingestion, there are plasma reductions in the ratio of tyrosine (Tyr) plus phenylalanine (Phe) to other large neutral amino acids (LNAA) with which Tyr and Phe compete for access to blood-brain barrier transporters (Boado et al, 1999). If Tyr and Phe are limited in their access to the brain, catecholamine synthesis is, in theory, inhibited. Evidence that DA neurotransmission is specifically impacted by the

\footnotetext{
*Correspondence: Dr M Luciana, Department of Psychology, University of Minnesota, N2 8 Elliott Hall, 75 East River Road, Minneapolis, MN 55455, USA, Tel: + I 612626 0757, Fax: + I 6126262079 , E-mail: lucia003@umn.edu

Received 21 February 2006; revised 7 June 2006; accepted 19 June 2006

Online publication: 27 June 2006 at http://www.acnp.org/citations/ Npp062706060 I20/default.pdf
}

manipulation is derived from animal, immunohistochemical, imaging, and human behavioral studies. This evidence will be briefly reviewed, since it supports the notion that ATPD primarily acts in limbic and striatal regions (areas known to modulate motivated behavior) and that as a consequence, ATPD subtly impairs response modulation during nonaffective tasks as well as affective processing.

Controlled animal studies demonstrate transient reductions of brain tyrosine but also DOPA (which reflects the extent to which DA synthesis is altered following precursor depletion) in cortical, striatal, and hippocampal regions following ATPD (Fernstrom and Fernstrom, 1995). Blunted responses to catecholamine ( $d$-amphetamine) challenge in tyrosine-depleted animals have also been reported and suggest that non-DA catecholamine systems are unaffected (McTavish et al, 1999a,b). In rats, $\left[{ }^{11} \mathrm{C}\right]$ Raclopride PET manipulations lead to reductions in blood and brain tyrosine as well as in brain DOPA and DA following ATPD. In this paradigm, an exogenous DA antagonist $\left(\left[{ }^{11} \mathrm{C}\right]\right.$ Raclopride $)$ is injected with the expectation that it will bind to available DA receptors. If DA synthesis is elevated prior to injection, available receptors should be bound with endogenous DA, and $\left[{ }^{11} \mathrm{C}\right]$ Raclopride will show decreased binding efficacy. However, if synthesis is decreased as should be the case following ATPD, available receptors will be unbound, and the exogenous compound will show increased binding efficacy. When $\left[{ }^{11} \mathrm{C}\right]$ Raclopride 
PET was used following ATPD, a slight (but not statistically significant) increase $(7 \%)$ in $\left[{ }^{11} \mathrm{C}\right]$ Raclopride binding was shown. However, following $d$-amphetamine injection, salinetreated control rats showed a significant reduction in $\left[{ }^{11} \mathrm{C}\right]$ Raclopride binding, while ATPD rats did not. This pattern suggests that ATPD significantly attenuates the increase in extracellular DA known to follow $d$-amphetamine administration (Le Masurier et al, 2004a). Similarly, when immunocytochemical detection of the early gene c-fos followed ATPD plus $d$-amphetamine administration, significant reductions in fos expression in depleted $v s$ control rats were found. The greatest differences were in the caudate, putamen, nucleus accumbens, bed nucleus of the stria terminalis and lateral habenula (Le Masurier et al, 2004b). The attenuated release of DA following $d$-amphetamine was not observed following ATPD without $d$-amphetamine, suggesting decreased regional production of DA but not changes in basal levels of extracellular DA following ATPD. Although these studies are not without pitfalls and raise questions about ATPD's efficacy to lower DA from baseline, it is notable that the areas most strongly impacted by the manipulation seem to be striatal and limbic regions.

Behavior is also altered in animals following ATPD. When the DA reuptake inhibitor cocaine, or the DA-releasing agent $d$-amphetamine were administered following ATPD in rats, the behavioral effects of those compounds were attenuated compared to patterns that followed a balanced amino-acid mixture, saline, a serotonin-releasing agent, or the direct DA receptor agonist apomorphine (McTavish et al, 2001a). Furthermore, ATPD decreased striatal DA release as measured by microdialysis following haloperidol administration and increased haloperidol-induce catalepsy, signifying reductions in DA availability (Jaskiw and Bongiovanni, 2004). In the only study to report use of this paradigm in non-human primates, ATPD in vervet monkeys reduced alcohol consumption compared a balanced mixture. No such reduction followed tryptophan depletion (Palmour et al, 1998). However, this study also reported a reduction of MHPG following ATPD, suggesting that the mixture's effects were not necessarily specific to DA given that MHPG is a major metabolite of norepinephrine.

Human studies suggest similar physiological effects as seen in rats. An early methodological study demonstrated significant reduction in blood TYR and PHE, as well as a significant decrease in systolic and diastolic blood pressure, suggestive of decreased catecholamine functioning following ATPD (Moja et al, 1996). In another study, the manipulation did not alter the evening secretion of melatonin from the pineal gland, suggesting that NE tone was unaffected (Sheehan et al, 1996). Human studies have repeatedly shown significant increases in serum prolactin following ATPD as compared to a balanced mixture, consistent with reduced hypothalamic DA activity (Harmer et al, 2001; Lythe et al, 2005; McTavish et al, 2004, 2005, 2001b). Furthermore, a study employing PET scanning following ATPD in humans showed that $\left[{ }^{11} \mathrm{C}\right]$ Raclopride binding in the striatum increased by six percent. Notably, the change in $\left[{ }^{11} \mathrm{C}\right]$ Raclopride binding correlated significantly $(r=-0.79)$ with the decrease in the blood TYR + PHE/LNAA ratio (Montgomery et al, 2003). Similarly, PET scanning during $d$-amphetamine challenge following ATPD in healthy males resulted in a significant increase in $\left[{ }^{11} \mathrm{C}\right]$ Raclopride binding (11.8\%), suggesting decreased DA release (Leyton et al, 2004). Interestingly, this study replicated the significant correlation between $\left[{ }^{11} \mathrm{C}\right]$ Raclopride binding efficacy and peripheral measures of precursor availability $(r=-0.82)$.

Despite this evidence that ATPD in humans is an efficacious method for lowering brain DA levels, its acute effects on human cognition and affective processing have yielded conflicting findings. Pharmacological challenges with DA receptor agonists and antagonists have suggested that spatial working memory processes are altered in response to systemic DA manipulations (Luciana and Collins, 1997; Mehta et al, 2001). This line of work was influential in suggesting targets for studies of ATPD's effects on cognition. Consistent with this literature, it was first reported that spatial working and recognition memory processes were adversely impacted by ATPD (Harmer et al, 2001; Harrison et al, 2004), leading to the notion that ATPD was nearly as efficacious as systemic drug ingestion in impacting executive cognitive processes. However, these same processes (using identical tasks) were unaffected in another study (Lythe et al, 2005). The reasons for these discrepancies are not known, although even conventional drug challenge studies have yielded conflicting patterns of cognitive reactivity following DA manipulations, perhaps due to the drugs' baseline dependent patterns of effects or other individual difference factors (see Mehta et al, 2001 for discussion). Recent studies have examined a broader array of cognitive and emotional functions that could be impacted by ATPD.

Previously depressed participants completed a rapid visual information processing task during ATPD $v s$ a balanced (BAL) condition (Roiser et al, 2005). ATPD resulted in significantly slower response times and reduced accuracy compared to BAL. In the same study, no differences in a One-Touch Tower of London task, a probabilistic reversal task, or a pattern-recognition memory task were found. However, ATPD reduced the size of wagers placed during a decision making task, suggestive of reduced risk-taking, reductions in reward salience, or increases in sensitivity to punishment. This finding, in conjunction with the decrement in attentional processing during the vigilance task, suggests that ATPD may exert effects distinct from the impairments in working memory that have typically been linked to cortical DA functioning (Luciana and Collins, 1997; Mehta et al, 2001). Indeed, current models that explain how and to what degree information processing is altered following ATPD might be somewhat narrow in focus given that the physiological studies reviewed above indicate that limbic and striatal functions might be the most appropriate targets for investigation.

Consistent with this idea, the literature suggests a circumscribed effect of ATPD on affective processing. One study compared the effects of ATPD, acute tryptophan depletion, and a BAL mixture on mood following public speaking and arithmetic challenges in healthy individuals (Leyton et al, 1999). The ATPD condition was associated with smaller increases in ratings of restlessness and larger decreases in self-reported liveliness compared to the control condition prior to stress challenge. Following the stress challenge tasks, ATPD participants continued to show larger decreases in liveliness and reported lowered mood 
via the elated-depressed scale of the POMS-bipolar form (Leyton et al, 1999). ATPD has also been shown to attenuate the emotional and behavioral effects of $d$-amphetamines in healthy humans, and it reduces symptoms of mania in bipolar individuals (McTavish et al, 2001b). ATPD in healthy adults has been reported to increase apathy on a novel visual-analog scale relative to individuals who ingest a BAL mixture. It also biases reaction times toward speedier response to negative words relative to positive words in an affective go/no-go task (McLean et al, 2004). This latter finding was replicated by Roiser et al (2005) at a trend level, in their study of recovered depressed individuals. In that study, ATPD did not impact self-reported apathy levels.

Overall, these studies suggest that ATPD attenuates mania and biases attention as individuals respond to affective stimuli, as reported by McLean and Roiser. Specifically, both investigators demonstrated that when individuals viewed affectively valenced words, they responded more quickly to negative than to positive words under the influence of ATPD. The difficulty of interpreting this latency bias (essentially a group by valence interaction) is that it cannot be readily characterized as an express deficit in processing positive words (ie, slower responses or difficulties in identifying positive stimuli) $v s$ facilitation in identifying or directing attention toward negative words. This distinction would be important to clarify in understanding the neural systems that are impacted by ATPD. Difficulties in categorizing and responding to positive words would be more consistent with anhedonia and suggestive of decreased DA activity in ventral striatal pathways (Depue and Iacono, 1989), while augmented responses to negative words might be more consistent with elevations in negative engagement as associated with dysregulation of noradrenergic or serotonergic systems (Harmer et al, 2003; Knutson et al, 1998). Indeed, if responses to both types of stimuli are impacted by the manipulation, then it may be the case that ATPD biases individuals toward a depressive state, which has been described in the personality literature as a decrement in positive affect (a feature that is specific to depressive illness) together with an augmentation of negative affect, which characterizes both depressive and anxiety disorders (Watson et al, 1988; Watson and Tellegen, 1985). It is also possible that ATPD alters inhibitory control processes in a manner that is independent of valence. In this study, our aim was to probe ATPD's effects on response inhibition and affective regulation in a more comprehensive manner. Healthy adults were studied. In order to expand upon the findings of McLean et al (2004), we included a neutral condition in an adaptation of the affective go/no-go task used by those researchers. Additionally, we also included a nonaffective go/no-go task. The go/no-go and affective go/no-go task were administered as part of a larger neuropsychological test battery (Luciana et al, 2005). Measures of motor speed and verbal fluency were also administered to rule out primary motor impairments as sources of decrements in performance attributed to ATPD.

\section{METHODS}

This study was approved by the university's Institutional Review Board. A total of 44 participants were recruited from undergraduate psychology courses and provided written, informed consent prior to completion of the study. They were compensated for their participation in the form of extra credit in a psychology course. In an initial screening interview, a medical and psychological history was obtained; participants were determined to be psychologically healthy using the Structured Clinical Interview for DSM-IV, patient version (First et al, 1997). A medical screening interview was administered, and exclusions were made for self-reported history of significant head trauma, neurological disease, daily nicotine use, recreational drug use ( $>1$ use per week), heavy alcohol use ( $>15$ drinks per week), current prescription medication use, current hormone contraceptive use, pregnancy, menstrual irregularities, and other conditions that might impact neural function. Any lifetime history of a DSM-IV Axis I disorder was grounds for exclusion. Participants were administered two subtests from the Wechsler Adult Intelligence Scale-3rd edition (WAIS-III: Wechsler, 1997), Vocabulary and Block Design, in order to estimate IQ They also completed selfreport personality questionnaires, including an abbreviated version of the Multidimensional Personality Questionnaire (MPQ-BF) (Patrick et al, 2002) and the Behavioral Inhibition System-Behavioral Activation System (BIS-BAS) questionnaire (Carver and White, 1994). Following this screening interview, participants were randomized in a double-blind fashion to either the ATPD or to a BAL. A daylong appointment was scheduled at the General Clinical Research Center (GCRC) for the amino-acid challenge procedure, hereafter referred to as the 'testing day.'

On the testing day, participants arrived at the GCRC at 0815 after following a low monoamine diet for $24 \mathrm{~h}$ and fasting from midnight the night before. Females were additionally required to complete the testing day during the follicular phase (between days 1 and 10) of the menstrual cycle. An indwelling cannula was inserted in the participant's nondominant arm at 0830 for blood draws at 0900 and 1400. Blood samples were collected at these times to obtain pre- and postmanipulation serum prolactin levels. Prolactin levels increase when central nervous system levels of DA decline (see Luciana and Collins, 1997 for a review). Due to funding constraints, amino acid levels were not obtained. At 0900 the amino acid beverage was consumed. Amino-acid mixtures were prepared as previously reported (Harmer et al, 2001; McTavish et al, 2001b). The ATPD beverage contained $15.0 \mathrm{~g}$ isoleucine, $22.5 \mathrm{~g}$ leucine, $17.5 \mathrm{~g}$ lysine, $5.0 \mathrm{~g}$ methionine, $17.5 \mathrm{~g}$ valine, $10 \mathrm{~g}$ threonine, and $2.5 \mathrm{~g}$ tryptophan. The BAL beverage was identical, except for the addition of $12.5 \mathrm{~g}$ Tyr and $12.5 \mathrm{~g}$ Phe. Female participants' beverages were reduced by $20 \%$ of these values due to their lower average body weight compared to males (this sample: $\mathrm{F}(1,36)=9.68, p<0.01$ ) (Harmer et al, 2001). Pharmaceutical-grade powdered amino acids purchased from Sigma-Aldrich (Saint Louis, MO) were mixed with 4.2 oz. Splenda ${ }^{\mathrm{TM}}$ and cherry-flavored Kool-aid ${ }^{\mathrm{TM}}$ in $300 \mathrm{ml}$ tap water. Participants were asked to consume the beverage using a straw within 5 min time, and afterwards were offered mint flavored gum and strong mints (Altoid ${ }^{\mathrm{TM}}$ brand) to reduce the unpleasant aftertaste. Neither the gum nor the mints contained phenylalanine though both contained small amounts of sugar; all participants elected to accept either gum or mints. 
Following consumption of the beverage, participants rested quietly but were prohibited from having visitors, talking on the phone, watching TV, using the internet or leaving the research center. At three time points throughout the day $(1100,1300$, and $1500 \mathrm{~h})$, fruit juice $(8 \mathrm{oz})$ was offered to prevent hypoglycemia. Blood pressure was monitored hourly throughout the study using an automated arm cuff. Following the second blood draw at $1400 \mathrm{~h}$ the cannula was removed. Cognitive testing began immediately thereafter and took $2 \mathrm{~h}$ to complete. Following the testing period, vital signs were recorded. Participants were then offered a snack and discharged from the research unit at approximately $1630 \mathrm{~h}$.

\section{Cognitive Tests}

Participants completed an Affective Go/No-Go task, a NonAffective Go/No-Go task (Braver et al, 2001), a Finger Tapping test (Reitan and Davison, 1974), and the Controlled Oral Word Association Test (COWAT) (Benton and Hamsher, 1976). The former two tasks were administered using the E-prime 1.1 (SP3) software and task presentation program (Psychological Software Tools, Pittsburg, PA) on a Dell Optiplex GX150 with Pentium III processor and 14-inch Dell monitor.

Affective go no-go. We employed an adaptation of the affective go/no-go used by Murphy et al (1999). This task is primarily a target detection task. Participants were presented with white words on a black background for $300 \mathrm{~ms}$. Each presentation was followed by a black screen to create an interstimulus interval of $900 \mathrm{~ms}$. In each of three blocks, participants were instructed to press the spacebar in response to positive, negative and neutral words, respectively, in that order. In each block, targets were presented with a frequency of $33 \%$; each block consisted of 60 trials. Words were selected from the Affective Norms for English Words (ANEW) database (Bradley and Lang, 1999) with the following characteristics: positive words had valence ratings greater than 7.0 on a bipolar scale of 1.0-9.0 where a score of ' 1.0 ' reflects maximum negative intensity, a score of ' 5.0 ' is neutral, and a score of ' 9.0 ' reflects maximum positive intensity. Using the same scale, negative words had valence ratings less than 3.0, and neutral words had valence ratings between 4.0 and 6.0. Additionally, positive and negative words were chosen so that subgroups of affective stimuli within each valence category varied in arousal level. High arousal words were characterized by arousal scores above 6.0 , and low arousal words were characterized by ratings below 5.5, also on a scale of 1.0-9.0. It was not possible to select neutrally valenced words that varied in arousal level. Although not part of the task's design a priori, word frequencies were determined to be matched across valences within each task block. Words were also matched in the number of syllables within and across each block of the task.

In addition to using different stimulus words and the incorporation of a block of neutrally valenced targets, this task differs from that used by Murphy et al (1999) and McLean et al (2004) in the number of task blocks and ordering of the blocks. Murphy et al (1999) used eight trial blocks that alternated every two blocks between happy $(\mathrm{H})$ and sad (S) targets (ie, HHSSHHSS).

Non-affective Go/No-Go task. On this task, participants were presented with white letters on a black background for $250 \mathrm{~ms}$ followed by presentation of a black screen for an intertrial interval of $1000 \mathrm{~ms}$ (Braver et al, 2001). During the first half of the task (No-Go block), participants were instructed to press the spacebar as quickly as possible for all letters except for the letter X. During the second half of the task (Target Detection), which resembles a traditionally defined continuous performance task except that it is shorter in duration, they were instructed to press the spacebar exclusively when presented with the letter X. Each portion was composed of 120 trials, with $20 \%$ X's.

For the Affective and Non-affective Go-No Go tasks, hit rate, false alarm rate, bias (c) and $\mathrm{d}^{\prime}$ were computed for each block. Reaction times were also recorded.

Finger tapping test. This task measures motor speed. Using a Finger Tapper board (Psychological Assessment Resources, Inc., Odessa, FL), participants tapped as rapidly as possible for three 10-s intervals. The right and left hands were assessed separately. The average number of taps across all trials, generated by each hand, was the primary variable of interest.

Controlled oral word association test (COWAT). The COWAT measures verbal fluency. Participants were instructed to name as many words as they could that begin with a given letter over 60 -s. Three letters (F, A, and S) were used. They were instructed to avoid generating proper nouns, numbers, and the same word multiple times with different endings. The COWAT is scored by subtracting perseverations and errors from the total number of words generated to yield the total number of correct responses for each letter.

\section{Assessment of Mood and Affect}

Participants completed mood measures administered at baseline (approximately 0840 hours) and hourly through 1500 hours including the Profiles of Mood States-Bipolar Form (POMS-Bi) (Lorr et al, 1982) and the Visual Analog Scale (VAS) (Bond and Lader, 1974); the Positive AffectNegative Affects Scales-Extended Version (PANAS-X) (Watson and Clark, 1994) was also administered at baseline and 1400 hours. The POMS-Bi is a 72 -item self-report questionnaire, which has been shown to be sensitive to subclinical changes in mood (Ellenbogen et al, 1996). It consists of six 12-item subscales: Composed/Anxious, Agreeable/Hostile, Elated/Depressed, Confident/Unsure, Energetic/Tired, Clearheaded/Confused. Raw scores on each subscale were transformed into T-scores using norms reported for college students (Lorr et al, 2003). Increasing scores reflect positive changes in mood, while decreases reflect negative mood states.

The VAS is a 14-item self-report scale, requiring the participant to make a mark on a $100 \mathrm{~mm}$ line between two opposing words (ie, Alert vs Drowsy) to indicate how they are feeling at that moment. Three factors have been 
described: Calmness, Alertness and Contentedness (Bond and Lader, 1974). The PANAS-X is comprised of the two unipolar scales, Negative Affect and Positive Affect, as well as 11 additional subscales: fear, hostility, guilt, sadness, joviality, self-assurance, attentiveness, shyness, fatigue, serenity, and surprise (Watson and Clark, 1994). Increases in PANAS-X subscale scores correspond to intensifications of that particular mood state or adjective rating.

\section{RESULTS}

Data were analyzed using the Statistical Package for the Social Sciences version 11.0 for Windows (SPSS Inc., Chicago, IL). Repeated measures, multivariate and oneway analyses of variance were performed when appropriate to the specific task. Nonparametric statistics were used in instances where data were non-normally distributed and appropriate transformations did not improve the skewness of the distributions and/or when other assumptions for parametric statistics were violated. Two-tailed tests are reported for all comparisons. Alpha levels below 0.05 were considered statistically significant.

\section{Demographic Differences between Groups}

After excluding participants who experienced nausea and vomiting as a side effect of the beverage (1 ATPD, 1 BAL) and participants whose testing was invalidated due to technical difficulties (4 ATPD, 1 BAL), the remaining participants $(n=16$ ATPD; $n=21$ BAL) did not differ between groups in gender, race, age, years of education, prorated WAIS IQ, weight or height (see Table 1). There were no significant differences between groups in selfreported personality traits as measured by the MPQ-BF $\mathrm{T}$-scores and the BIS/BAS.

\section{Effect of ATPD on Serum Prolactin Secretion (Figure 1)}

Valid prolactin values before and after tyrosine depletion were available for a subgroup of those tested. Four individuals (3 ATPD, $1 \mathrm{BAL}$ ) were excluded from this analysis because they had out-of-range predrink values for the assay used, which we attribute to anxiety (Torner et al,

Table I Participant Characteristics by Condition

\begin{tabular}{lcc}
\hline & \multicolumn{2}{c}{ Condition } \\
\cline { 2 - 3 } Variable & ATPD & BAL \\
\hline$N$ & 16 & 21 \\
Number of male:female & $10: 6$ & $14: 7$ \\
Number of Caucasian:Minority & $15: 1$ & $19: 2$ \\
Age (years) & $19.50 \pm 1.03$ & $18.90 \pm 1.14$ \\
Prorated WAIS-III IQ & $120.80 \pm 10.99$ & $119.40 \pm 9.08$ \\
Height (m) & $1.73 \pm 0.13$ & $1.78 \pm 0.08$ \\
Weight (kg) & $71.49 \pm 12.24$ & $71.42 \pm 11.92$ \\
Years of schooling completed & $13.13 \pm 0.89$ & $12.86 \pm 0.96$
\end{tabular}

Unless otherwise noted, all values represent means \pm I SD.
2004). Log-transformed pre- $v s$ postbeverage prolactin levels were examined in a repeated measures analysis of variance with Time as a within-groups factor and Condition as a between-groups factor. There was a significant main effect of Condition $(\mathrm{F}(1,31)=12.73, p=0.001)$, but no significant main effect of Time $[\mathrm{F}(1,31)=0.002$, NS]. There was a significant Condition by Time interaction $(\mathrm{F}(1,31)=6.42, p<0.05)$. Post hoc one-way analyses of variance showed that plasma prolactin did not differ significantly between conditions at $0900(\mathrm{~F}(1,31)=2.40$, NS), but prolactin level for those in the ATPD group was significantly higher than for BAL at $1400(\mathrm{~F}(1,31)=28.32$, $p<0.001)$.

\section{Mood Measures (Table 2)}

In our analyses of the mood measures, we focused on ratings provided at two time points: 0845 (baseline) and 1400 (immediately pretesting). For baseline to pretesting, a repeated measures analysis of variance test was conducted for each mood variable, with that Variable as the withinsubjects factor and Condition as the between-subjects factor. All results for the POMS-Bi scales showed the same pattern of significance regardless of whether raw scores or normed T-scores were analyzed; T-scores are reported herein. Table 2 summarizes these findings. While many measures showed main effects of Time across drink conditions, no measure showed a main effect of Condition. On the VAS, summed scores across all items yielded a trend toward a Condition $\times$ Time interaction $(\mathrm{F}(1,35)=3.25$, $p<0.10$ ) but the only (marginally) significant interaction at the item or factor level was for the Alert scale $(p=0.10)$. Contrary to expectation, individuals in the ATPD condition reported increased alertness $5 \mathrm{~h}$ after testing as compared to baseline $(p<0.01)$, while no significant change was evident in the BAL group. There was also a Condition $\times$ Time interaction for the PANAS-X hostility scale $(\mathrm{F}(1,35)=5.46$, $p<0.05)$ with individuals in the ATPD group reporting significant increases in self-reported hostility, while individuals in the BAL group were unchanged from baseline (Table 2).

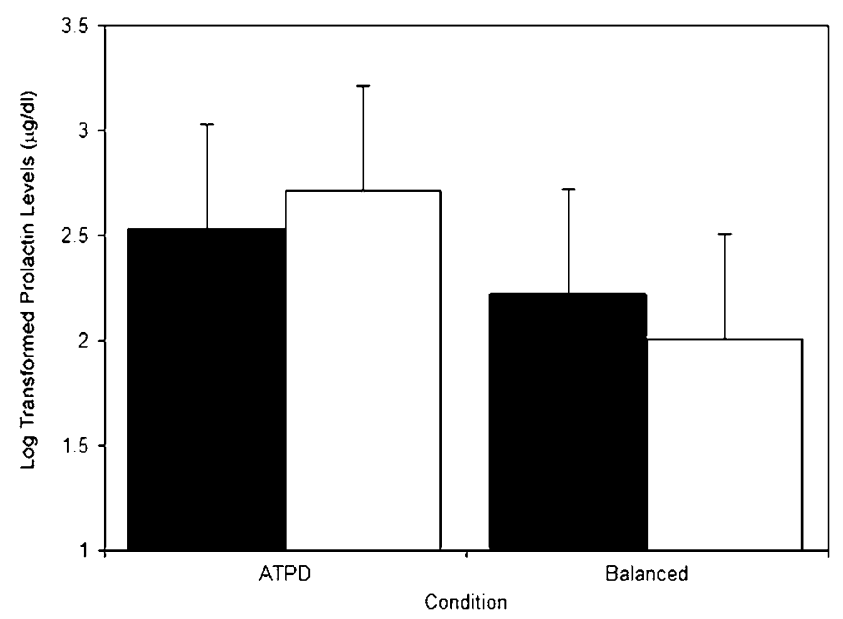

Figure I Plasma prolactin by time and condition. Plasma prolactin values (log-transformed) predrink (ם) vs-postdrink ( $\square$ ) by condition. As explained in the text, the groups varied in their postdrink values. 
Table 2 Summary of Mood Rating Effects Baseline to Pretesting

\begin{tabular}{|c|c|c|c|}
\hline Variable & $\begin{array}{l}\text { Effect of } \\
\text { time }\end{array}$ & $\begin{array}{l}\text { Effect of } \\
\text { condition }\end{array}$ & $\begin{array}{l}\text { Condition } \times \text { time } \\
\text { interaction }\end{array}$ \\
\hline \multicolumn{4}{|l|}{ POMS-Bi Scale } \\
\hline A. Composed/Anxious & - & - & - \\
\hline B. Agreeable/Hostile & Decrease* & - & - \\
\hline C. Elated/Depressed & - & - & - \\
\hline D. Confident/Unsure & - & - & - \\
\hline E. Energetic/Tired & Increase ${ }^{* *}$ & - & - \\
\hline $\begin{array}{l}\text { F. Clearheaded/ } \\
\text { Confused }\end{array}$ & - & - & - \\
\hline \multicolumn{4}{|l|}{ Visual Analog Scale (VAS) } \\
\hline Calmness & Increase ${ }^{* *}$ & - & - \\
\hline Alertness & Increase ${ }^{* *}$ & - & $\begin{array}{l}\text { Increase for ATPD } \\
\text { v.BAL** }\end{array}$ \\
\hline Contentedness & Increase* & - & - \\
\hline \multicolumn{4}{|l|}{ PANAS-X } \\
\hline Positive affect & - & - & - \\
\hline Negative affect & Decrease*** & - & - \\
\hline Fear & Decrease*** & - & - \\
\hline Hostility & - & - & $\begin{array}{l}\text { Increase for ATPD } \\
\text { v.BAL*** }\end{array}$ \\
\hline Guilt & - & - & - \\
\hline Sadness & - & - & - \\
\hline Joviality & - & - & - \\
\hline Self-assurance & Decrease*** & - & - \\
\hline Attentiveness & Increase* & - & - \\
\hline Shyness & Decrease*** & - & - \\
\hline Fatigue & Decrease*** & - & - \\
\hline Serenity & Increase** & - & - \\
\hline Surprise & Decrease*** & - & - \\
\hline
\end{tabular}

一, no difference, ${ }^{*} p<0.10$, *** $p<0.05$.

\section{Cognitive Tests}

Affective Go/No-Go. Means and standard deviations for all cognitive tests are provided in Table 3. Our strategy in analyzing data from this task was to first assess whether we could replicate the findings of McLean et al (2004) and Roiser et al (2005) with respect to differences between detection of positive $v s$ negative stimuli. We then extended their work by considered whether comparisons involving the neutral condition could clarify our interpretations. Findings are generally summarized in Table 4, which indicates comparisons that were and were not significant. Below, we highlight significant effects. We computed $d^{\prime}$, a bias measure (c), hit rate, and false alarm rates for each task.

$d^{\prime}$, an index of the participant's ability to differentiate signal (target) from noise (nontargets) that corrects hit rate for false alarm rate, was calculated. When positive and negative valence categories were compared, there was no main effect of Valence, nor was there a main effect of Condition. There was a trend toward a Condition by
Valence interaction $[\mathrm{F}(2,34)=3.40, p<0.10]$. Post hoc analyses revealed that the individuals in the ATPD vs BAL group had marginally lower $d^{\prime}$ scores for positive words $(\mathrm{F}(1,36)=3.62, p<0.10)$ but were not different from the BAL condition in detecting negative words. Responses to neutral words did not vary between groups (see Figure 2, top panel). $d^{\prime}$ did not vary when neutral words were compared to positive words or when neutral words were compared to negative words between groups.

Bias, an index of the decision criterion that an individual uses to distinguish signals from noise in such tasks, was calculated using $c$ (Stanislaw and Todorov, 1999). When positive and negative blocks were compared, there was a main effect of Valence $(\mathrm{F}(1,35)=5.90, p<0.05)$ with higher criteria used to make decisions about positive stimuli. However, there were no effects or interactions by Condition. Similar valence effects were observed when responses to positive words were compared to neutral (a higher criterion for decisions involving positive words) and when responses to neutral words were compared to negative words (a higher criterion for decisions to negative words).

The components of $d^{\prime}$ (hit rate and false alarm rate) were analyzed similarly. When hit rate was examined between positive and negative valence blocks, there was a main effect of Valence $(\mathrm{F}(1,35)=21.45, p<0.001)$ as well as a significant Condition $\times$ Valence interaction $(\mathrm{F}(1,35)=4.149$, $p<0.05)$. Hit rate was higher for negative relative to positive words, but more strongly so for individuals in the ATPD $(\mathrm{F}(1,15)=18.18, p=0.001)$ vs the BAL condition $(\mathrm{F}(1,20)=4.14, p=0.06)$, as indicated in the middle panel of Figure 2. Examination of this figure suggests that individuals in the ATPD condition were slightly worse at detecting positive words but slightly better at detecting negative words relative to individuals in the BAL condition; however, one-way analyses of variance to examine hit rates between groups yielded no significant differences in either measure between groups.

When the hit rate was compared between neutral and positive words, there were no differences by Condition or a Condition $\times$ Valence interaction. When hit rates for neutral words were compared to those for negative words, there was a trend toward a significant Condition $\times$ Valence interaction $(\mathrm{F}(1,35)=2.90, p<0.10)$. Within the BAL group, the hit rate to negative words was significantly lower relative to neutral words $(\mathrm{F}(1,20)=4.96, p<0.05)$. Within the ATPD group, there was no significant difference in hit rate for negative relative to neutral words.

Thus, if we interpret the findings observed in the BAL group as an indicator of how responses would be patterned under placebo conditions, the hit rate to negative words appears to be augmented in the ATPD group relative to both positive words and to neutral words.

To determine whether these findings were influenced by word arousal level, we examined hit rates to positive relative to negative words using arousal (hit rate to low vs high arousal words) as a second within-groups factor. Although high arousal words were easier to identify $(F(1,35)=37.9$, $p<0.001$ ), there was no interaction between Arousal level and Condition.

Arousal levels did not vary among neutral words or when neutral words were compared to positive and negative words. 
Table 3 Cognitive Task Performance by Condition

\begin{tabular}{|c|c|c|}
\hline Condition variable & ATPD & BAL \\
\hline \multicolumn{3}{|l|}{ Affective Go/No-Go task } \\
\hline \multicolumn{3}{|l|}{$d^{\prime}$ (sensitivity) } \\
\hline Positive & $3.83 \pm 1.76$ & $5.35 \pm 2.82$ \\
\hline Neutral & $3.14 \pm 2.11$ & $3.37 \pm 1.96$ \\
\hline Negative & $4.80 \pm 2.08$ & $4.83 \pm 2.13$ \\
\hline \multicolumn{3}{|l|}{ c (bias) } \\
\hline Positive & $1.09 \pm 0.97$ & $1.24 \pm 1.04$ \\
\hline Neutral & $-0.64 \pm 0.78$ & $-0.71 \pm 0.90$ \\
\hline Negative & $0.35 \pm 1.30$ & $0.78 \pm 1.29$ \\
\hline \multicolumn{3}{|l|}{ Hit rate } \\
\hline Positive & $0.77 \pm 0.16$ & $0.81 \pm 0.13$ \\
\hline Neutral & $0.91 \pm 0.08$ & $0.92 \pm 0.07$ \\
\hline Negative & $0.91 \pm 0.08$ & $0.86 \pm 0.10$ \\
\hline \multicolumn{3}{|l|}{ False alarm rate } \\
\hline Positive & $0.03 \pm 0.04$ & $0.04 \pm 0.07$ \\
\hline Neutral & $0.20 \pm 0.12$ & $0.18 \pm 0.11$ \\
\hline Negative & $0.04 \pm 0.04$ & $0.03 \pm 0.04$ \\
\hline \multicolumn{3}{|l|}{ Reaction time (ms) } \\
\hline Positive & $308.35 \pm 68.75$ & $299.24 \pm 59.45$ \\
\hline Neutral & $312.80 \pm 85.89$ & $318.89 \pm 82.83$ \\
\hline Negative & $293.00 \pm 62.86$ & $305.68 \pm 72.59$ \\
\hline
\end{tabular}

Go/No-Go task

No-Go portion

$d^{\prime}$

$2.15 \pm 1.08$

$-0.37 \pm 0.49$

Hit rate

False alarm rate

Reaction time (ms)

$0.88 \pm 0.09$

$0.25 \pm 0.08$

$79.07 \pm 40.98$

Percent omissions I after $X$

Percent omissions 2 after $X$

Percent omissions 3 after $X$

Percent omissions $4+$ after $X$

$31.09 \pm 29.17$

$9.95 \pm 8.51$

$|3.33 \pm| 0.6 \mid$

$39.38 \pm 29.58$

Target detection portion

$\begin{array}{lrc}d^{\prime} & 8.89 \pm 1.76 & 8.65 \pm 1.58 \\ c & -0.13 \pm 0.64 & 0.07 \pm 1.02 \\ \text { Hit rate } & 0.99 \pm 0.01 & 0.98 \pm 0.08 \\ \text { False alarm rate } & 0.003 \pm 0.005 & 0.003 \pm 0.005 \\ \text { Reaction time } & 141.12 \pm 48.65 & 150.26 \pm 52.74\end{array}$

Controlled oral word association test (number of correct responses)

$\mathrm{F}$

A

$11.50 \pm 4.21$

$11.19 \pm 3.93$

$15.00 \pm 3.62$
$14.43 \pm 3.47$

$12.10 \pm 3.85$

$3.04+1.52$

$-0.63 \pm 0.49$

$0.94 \pm 0.08$

$0.21 \pm 0.11$

$108.61 \pm 57.28$

$8.78 \pm 14.59$

$11.29 \pm 14.5$

$15.50 \pm 17.23$

$50.15 \pm 33.16$

$16.05 \pm 4.70$
Table 3 Continued

\begin{tabular}{lcc}
\hline Condition variable & ATPD & BAL \\
\hline $\begin{array}{l}\text { Finger tapping test (number of taps) } \\
\text { Dominant hand }\end{array}$ & \\
$\quad$ Trial I & $42.00 \pm \mid 1.86$ & $41.90 \pm 13.53$ \\
Trial 2 & $47.94 \pm \mid 1.98$ & $45.76 \pm 13.5 \mid$ \\
Trial 3 & $48.38 \pm 10.87$ & $46.8| \pm| 1.66$ \\
& & \\
Nondominant hand & & \\
Trial I & $43.06 \pm 8.82$ & $41.95 \pm 13.56$ \\
$\quad$ Trial 2 & $44.00 \pm 8.47$ & $42.29 \pm 12.03$ \\
Trial 3 & $42.50 \pm 8.19$ & $43.86 \pm 9.4 \mid$ \\
\hline
\end{tabular}

All values represent means \pm I SD.

Table 4 Summary of Affective Go No-Go Task Findings

\begin{tabular}{|c|c|c|c|}
\hline & Condition & Valence & $\begin{array}{l}\text { Condition } \times \\
\text { Valence }\end{array}$ \\
\hline$d^{\prime}$ : Positive vs Negative & - & - & Trend \\
\hline$d^{\prime}:$ Neutral vs Negative & - & Significant & - \\
\hline$d^{\prime}$ : Neutral vs Positive & - & Significant & - \\
\hline c: Positive vs Negative & - & Significant & - \\
\hline c: Neutral vs Negative & - & Significant & - \\
\hline c: Neutral vs Positive & - & Significant & - \\
\hline Hit rate: Positive vs Negative & - & - & Significant \\
\hline Hit rate: Neutral vs Negative & - & - & Trend \\
\hline Hit rate: Neutral vs Positive & - & Significant & - \\
\hline False alarm rate: Positive vs Negative & - & - & - \\
\hline False alarm rate: Neutral vs Negative & - & Significant & - \\
\hline False alarm rate: Neutral vs Positive & - & Significant & - \\
\hline Reaction time: Positive vs Negative & - & - & - \\
\hline Reaction time: Neutral vs Negative & - & - & - \\
\hline Reaction time: Neutral vs Positive & - & - & - \\
\hline Efficiency: Positive vs Negative & - & - & Significant \\
\hline Efficiency: Neutral vs Negative & - & - & - \\
\hline Efficiency: Neutral vs Positive & - & - & - \\
\hline
\end{tabular}

False alarm rate was analyzed as described above. There were no main effects or interactions by Condition (see Figure 2, bottom panel and Table 4). False alarm rates were comparably higher when participants were instructed to respond to neutral words. Whether errors of commission were made preferentially to positive $v s$ negative words was examined (ie, did participants tend to classify positive words as 'neutral'?). Errors of commission in the neutral condition were not systematic in any way that could be statistically determined.

A reaction time or latency bias representing faster reaction times to negative words $v s$ positive words following ATPD was previously reported (McLean et al, 2004). To replicate this finding, we compared the reaction times to positive $v s$ negative words between groups. Although the 

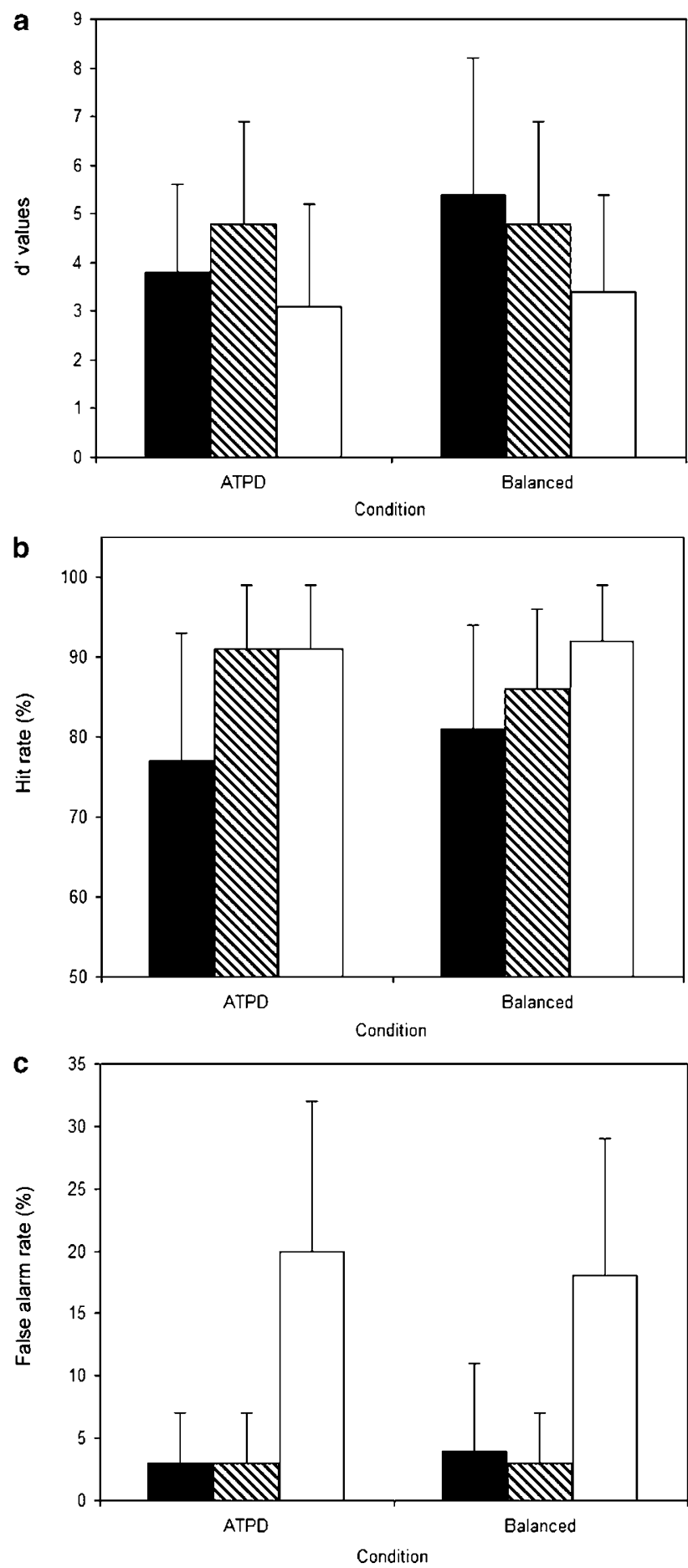

Figure 2 Affective Go/No-Go performance by valence and task condition. (a) $d^{\prime}$ scores for blocks where participants were instructed to respond to positive $(\boldsymbol{\square})$, negative (N), or neutral words $(\square)$, respectively. (b) Hit rates. (c) False alarm rates.

groups descriptively differed in a manner consistent with what has previously been reported (ATPD individuals showed slower responses to positive and faster responses to negative words), this analysis yielded no significant main effects of Condition or Valence, and the Condition by Valence interaction eluded statistical significance $(\mathrm{F}(1,36)=$ $2.65, p>0.10)$. In addition, no significant differences in

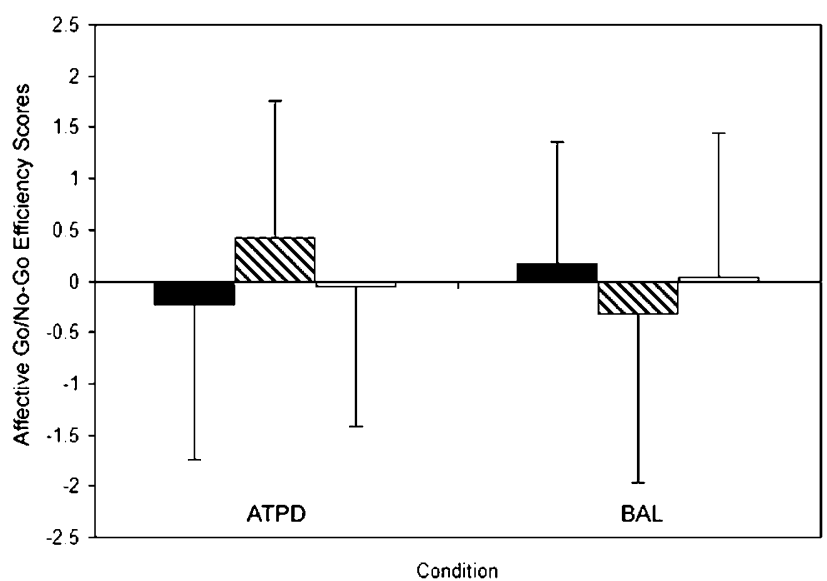

Figure 3 Efficiency scores for affective Go/No-Go by condition. affective Go No-Go efficiency scores for positive $(\boldsymbol{\square})$, negative ( $\mathbb{R}$ ), and neutral $(\square)$ were created by summing standardized reaction time and hit rate scores. As indicated in the graph, individuals in the ATPD group show decreased efficiency in responding to positive relative to negative words without differences in their responses to neutral words.

reaction times to neutral $v s$ positive or neutral $v s$ negative words were found. Reaction times were not significantly correlated with hit rates, false alarm rates, $c$, or $d^{\prime}$ scores for either group within each valence category.

Finally, an efficiency score was created that combined the hit rate with reaction times to stimuli within each valence category. Hit rates and reaction times were first $z$ transformed, then scaled so that high scores represented faster (reaction times) and more accurate (hit rate) performance. These standard scores were then summed for each participant. Levels above 0 reflect greater efficiency in responding to a stimulus set; levels below 0 reflect decreased processing efficiency. Efficiency scores for positive $v s$ negative stimuli were compared between groups yielding no significant main effects of Valence or Condition but a highly significant Valence $\times$ Condition interaction $(\mathrm{F}(1,35)=7.92, p<0.001)$. As indicated in Figure 3, the relative efficiency of evaluating negative cues was enhanced by ATPD, while the relative efficiency of evaluating positive cues appears to be decreased. The important variable is the relative distance from one another occupied by each affective process. Oneway analyses of variance did not yield significant between-group differences in efficiency scores, while within-group analyses supported the notion that the valences were distinct in the ATPD $(\mathrm{F}(1,15)=6.99, p<0.05)$ but not in the BAL $(F(1,20)=2.70$, NS) condition. The efficiency score for neutral words was unaffected by condition. There were no main effects or interactions by Condition when efficiency scores to neutral words were compared to those for positive or to negative words.

To summarize, if the BAL condition serves as a true placebo, then this overall pattern of findings suggests that ATPD served to sensitize responses to negative words, while at the same time depressing the processing of positive words. Which process is maximally affected by the manipulation cannot be determined within our sample. The responses to neutral words alone or relative to the other valence conditions appears to be unaffected by the manipulation. 
Nonaffective Go/No-Go. One question raised by the previous analysis concerns the specificity of findings to a response control task that has an affective component. To determine between-group differences on a nonaffective task measuring target detection and response inhibition ability, $d^{\prime}$, bias scores, hit rates, false alarm rates, and reaction times for both the target detection and no-go blocks were examined using Condition as the between groups factor. Due to some variables having skewed distributions even after transformations were applied, the Mann-Whitney $U$ nonparametric procedure was used for these analyses.

Within the Target Detection block, there were no significant group differences in $d^{\prime}(U=149.0, \mathrm{NS})$, bias scores $(U=161.0, \mathrm{NS})$, hit rate $(U=153.5, \mathrm{NS})$, false alarm rate $(U=163.0, \mathrm{NS})$, or reaction time $(U=146.0, \mathrm{NS})$.

Within the No-Go block, individuals in the ATPD group had lower $d^{\prime}$ scores than those in the BAL group $(U=80.0$, $p<0.01$ ), which appears to be driven by ATPD's significantly lower hit rate than BAL $(U=78.5, p<0.01)$ rather than by false alarm rate, which did not differ between groups $(U=139.5$, NS). Bias differed between groups $(U=105.0$, $p=0.05)$ with individuals in the ATPD condition having higher scores. Those in the ATPD group also demonstrated faster responses than BAL $(U=78.0, p<0.01)$.

Examinations of the speed-accuracy tradeoff within each group indicated that for individuals in the ATPD condition, hit rate and reaction time were positively correlated (Spearman's $r=0.61, p<0.05$ ) such that a higher hit rate was associated with slower response times. Hit rate is low when correct responses are omitted, and on this portion of the task, the task is designed to yield responses most of the time (80\% targets). Omission errors are typically considered to represent attentional lapses, which (unlike the pattern observed here) might be expected to be associated with slower $v s$ faster responses.

In contrast, response times did not significantly relate to false alarms (Spearman's $r=-0.29$, NS) suggesting that individuals in the ATPD condition were not more impulsive in a conventional sense. Thus, within the ATPD condition, more deliberate (slower) responding is associated with better maintenance of attention and fewer omission errors. In contrast, faster responding is associated with attentional failures, perhaps suggesting motivational failures.

For individuals in the BAL condition, hit rate was not significantly correlated with response times (Spearman's $r=0.23$, NS), but it was related to false alarm rate (Spearman's $r=-0.50, p<0.05$ ) such that a high number of false alarms was associated with faster, perhaps more impulsive, responses. Thus, the groups differed in how the timing of their responses aided (or negatively impacted) performance on this task.

It must be emphasized that faster responses cannot fully account for the error patterns generated by individuals in the ATPD group. We conducted a post hoc examination of whether there were any patterns to the errors of omission made by individuals in the ATPD condition during the NoGo block. One hypothesis was that the errors of omission made by those in the ATPD group, which resulted in lower hit rates compared to BAL, may have occurred systematically following presentation of the letter ' $\mathrm{X}$,' for which participants were to inhibit a response. To test this hypothesis, we coded all the stimuli presented within three letters following the presentation of ' $X$ ' as ' 1 after $X$,' '2 after X,' ' 3 after X,' and 'four or more after X.' Because the order of stimulus presentation was randomly generated by the task, it was important to verify that there were not systematically different opportunities between groups to make responses following presentations of $\mathrm{X}$. Mann-Whitney U's with Condition as the between-groups factor showed that there were statistically equivalent opportunities for responses (and thus errors) in both groups, with the exception of a trend toward a greater number of letters presented 1 after $X$ for the ATPD group than the BAL group $(U=117.0, p=0.09)$. Although this difference is marginally statistically significant, the actual means are, for all practical purposes, equivalent between groups. In the BAL condition, individuals were presented with $31.6 \pm 0.63$ letters immediately following $X$ with a range $=30-32$ letters. In the ATPD condition, individuals were presented with $31.2 \pm 0.68$ letters following $\mathrm{X}$, with an equivalent range of 30-32 letters. There were no group differences in opportunities to respond to letters 2 after X $(U=144.0, \mathrm{NS}), 3$ after X $(U=143.5, \mathrm{NS})$, or four or more after $\mathrm{X}(U=129.5, \mathrm{NS}))$.

The percentage of errors of omission at 1 after $\mathrm{X} v s$ all other locations were then analyzed using a repeated measures analysis of variance, with Proximity to $\mathrm{X}$ as a within groups factor, and Condition as the between-groups factor. Significance levels are reported here using the Huynh-Feldt correction. There was a significant main effect of Proximity to $\mathrm{X}(\mathrm{F}(1,35)=39.41, p<0.001)$, no main effect of Condition $(\mathrm{F}(1,35)=0.59, \mathrm{NS})$, and a significant interaction of Proximity to $\mathrm{X}$ with Condition $(\mathrm{F}(1,35)=5.31$, $p<0.05)$.

Post hoc analyses using nonparametric procedures were performed to follow-up on the repeated measures analysis. Nonparametric procedures were used, because transformations did not equalize the variance between groups for all variables. Mann-Whitney $U$ comparisons revealed that those in the ATPD group had a significantly greater proportion of their errors of omission for the stimuli immediately following presentation of $\mathrm{X}$ compared to BAL $(U=65.0, p=0.001)$, but no significant difference in proportions of omissions for stimuli two, three, or fourplus presentations after $\mathrm{X}(U=156, \mathrm{NS} ; U=164.5, \mathrm{NS}$, $U=138$, NS) (Figure 4). Thus, individuals in the ATPD condition may have experienced a subtle and transient impairment in the ability to re-initiate responding following the demand to inhibit responses, a finding which has not been previously reported in the ATPD literature.

Finger tapping test. Data were analyzed using a repeated measures analysis of variance, with Trial and Hand (dominant $v s$ nondominant) as within groups factors, and Condition as the between group factor. There was a main effect of Hand, such that individuals generated more taps with the dominant hand $(\mathrm{F}(1,35)=5.41, p<0.05)$. There was also a main effect of Trial, with successive trials yielding greater taps $(\mathrm{F}(2,70)=7.27, p<0.01)$. The interaction of Hand by Trial was significant $(\mathrm{F}(2,70)=8.91, p<0.001)$ such that taps increased significantly across successive trials in the dominant hand but not in the nondominant hand. There were no main effects of Condition or additional interactions. 


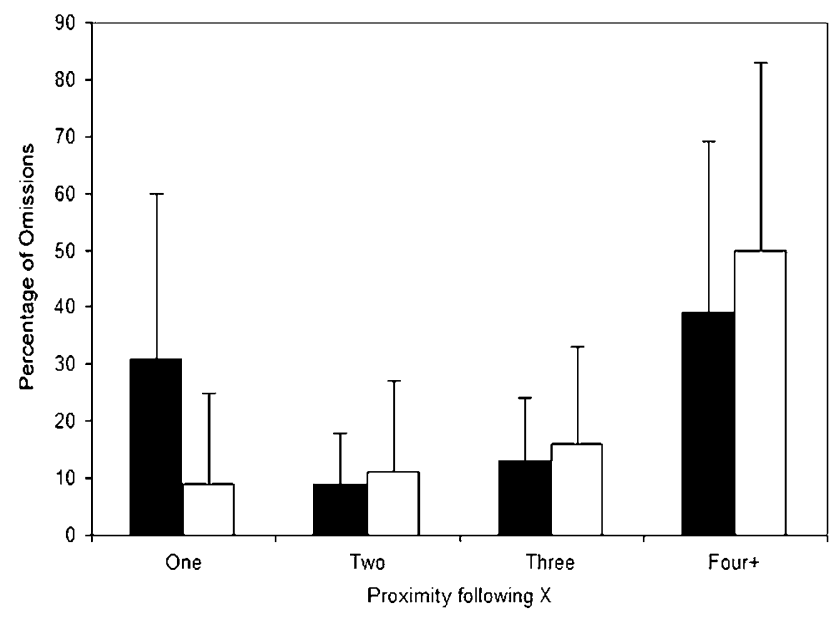

Figure 4 Errors of omission following demand for inhibitory control: nonaffective Go/No-Go. Percent errors of omission on the nonaffective go no-go task. Scores were calculated for omission errors that occurred one, two, three, or four-plus stimuli following an $X$ (a demand for response inhibition). Individuals in the ATPD group ( $\mathbf{\square}$ ) made more omission errors immediately following an $\mathrm{X}$ than did the BAL group $(\square)$.

Controlled oral word association test (COWAT). In a repeated measures analysis of variance using number of words generated for each Letter (F, A, and S) as the withingroups factor, and Condition as the between-groups factor, there was a main effect of Letter $(\mathrm{F}(2,70)=15.38, p<0.001)$, such that Letter 2 (A) had significantly lower correct words produced than Letter $1(\mathrm{~F})(t(36)=2.08, p<0.05)$ or Letter 3 (S) $(t(36)=5.27, p<0.001)$. Letter $1(\mathrm{~F})$ had significantly lower correct words produced than Letter 3 (S) $(t(36)=3.57, p=0.001)$. There was no significant main effect of Condition $(\mathrm{F}(1,35)=2.94$, NS) or an interaction of Letter by Condition $(\mathrm{F}(2,70)=1.26$, NS). Although this interaction was not significant, the findings from the go nogo task prompted us to conduct several post hoc explorations of error patterns on this task to assess whether task initiation or responses to stop-start demands were distinct between groups. We found that ATPD individuals produced significantly fewer correct words during the first trial than those in the BAL condition $(\mathrm{F}(1,35)=5.37, p<0.05)$. The groups did not differ in the number of perseverations or rule-breaking errors on this task.

Associations among ATPD responses, mood, and behavior. Prolactin responses to ATPD and BAL mixtures were calculated (postdrink prolactin-predrink prolactin) and associated with mood and cognitive changes. These associations were evaluated for individuals in the ATPD group who generated valid prolactin data using Spearman rank-order correlations. No significant correlations were observed between prolactin responses and mood, prolactin responses and cognitive performance, or prolactin responses and personality traits as measured by the MPQ and BIS/BAS scales.

Personality traits (BIS/BAS and MPQ) were also examined in relation to cognitive and mood reactivity to ATPD focusing on variables that showed significant or nearsignificant alternations with ATPD. These variables included alertness ratings on the VAS, ratings of hostility on the PANAS-X, Affective Go-No Go $d^{\prime}$ scores for pleasant minus unpleasant stimuli, hit rates for pleasant minus unpleasant stimuli, reaction times for pleasant minus unpleasant stimuli, efficiency scores for pleasant minus unpleasant stimuli, plus hit rates, bias, reaction times, and omission errors one trial after $\mathrm{X}$ on the go no-go task.

On the affective go no-go task, the only near-significant correlation was that individuals with lower BIS scores on the BIS/BAS showed a relatively low hit rate to positive and a high hit rate to negative words on the affective go no-go task $(r=0.45, p<0.10)$.

For the nonaffective go no-go task, slower no-go reaction times were associated with higher MPQ Constraint scores $(r=0.80, p=0.000)$. Higher bias scores were associated with higher MPQ negative emotionality scores $(r=0.44, p<0.10)$ and with lower MPQ Constraint scores $(r=-0.55, p<0.05)$. Lower hit rates were associated with higher negative emotionality scores $(r=-0.53, p<0.05)$, with lower Constraint scores $(r=0.55, p<0.05)$, and at a trend level with higher BAS drive scores $(r=-0.49, p<0.10)$. A higher percentage of omission errors one trial after $\mathrm{X}$ was associated with lower MPQ Positive Emotionality scores $(r=-0.50, p=0.05)$.

\section{DISCUSSION}

We conducted a randomized, double-blind, betweengroups investigation of cognitive and affective functioning in healthy adults following consumption of a tyrosinephenylalanine depleted beverage or a balanced control beverage.

Effective depletion of hypothalamic DA levels in ATPD is supported by our finding of significantly higher serum prolactin levels in the ATPD vs BAL group $5 \mathrm{~h}$ after aminoacid ingestion. Decreases in prolactin levels throughout the day were observed for individuals in the BAL condition and are consistent with the reported diurnal rhythms of prolactin (for a review see Freeman et al, 2000). Thus, it appears that DA levels were altered in the central nervous system as a consequence of our manipulation in a manner that is consistent with DA depletion. Although our prolactin effect is not large in magnitude, it compares favorably to findings reported by other labs that have measured PRL responses to ATPD (Harmer et al, 2001; Lythe et al, 2005; McTavish et al, 2004, 2005, 2001b). When we quantified degree of prolactin change from these other studies, we calculated an average post-ATPD increase in prolactin of $25.24 \%$, with a range from -7 to $50 \%$, depending on the report. Only one study (Lythe et al, 2005) reported a decrease in PRL levels following ATPD and in that report, the decrease was less than what was observed following ingestion of a BAL mixture, suggesting that ATPD blunted the diurnal decrease that was expected. We observed a mean increase of $16.6 \%$ in PRL levels following ATPD, which is well within the range that others have reported. Our data also conform to what others have reported regarding decreases in prolactin levels after ingestion of the balanced mixture (reported ranges $=$ decreases of $35.2-15.5 \%$; our decrease is $19.2 \%)$. Therefore, it appears that ATPD exerted the predictable antagonist effect on brain hypothalamic DA systems. Beyond that, we report three main findings. 
Our first major finding is we observed no mood changes in our participants as a consequence of the ATPD manipulation despite our assessment of mood state using several well-validated instruments. We failed to replicate others' (McLean et al, 2004) reports of increased apathy in healthy adults following ATPD. However, it should be emphasized that we did not employ the same apathy scale used by McLean and co-workers. In addition, we did observe that regardless of condition, individuals reported an overall tendency toward decreases in negative affect over time, while positive affect was overall unchanged. It appears that individuals begin the day with some trepidation, which resolves as the day goes on. As to why no changes in positive affect were observed, it may be that the highly structured, relatively unstimulating, and physically aversive nature of the study day overcomes any subtle change in mood that might otherwise be specifically observed as a consequence of the ATPD manipulation, at least in psychologically healthy individuals. An increase in alertness over time, based on the VAS, interacted with drug condition $(p=0.10)$ with elevations in alertness during ATPD, but the significance of this finding is unclear given the number of statistical comparisons that we conducted. Individuals in the ATPD group also reported increased hostility.

Our second finding is that in an affective target detection task, ATPD yielded a subtle pattern of responses that suggests a heightened state of attention to negative words in the context of a diminished sensitivity to positive words. There was an increased ability to detect negative relative to positive or neutral words that was driven by hit rate as opposed to false alarms for those in the ATPD condition. In addition, although the findings were not statistically significant, we observed reaction times that were faster to negative vs positive words during ATPD, as has been reported in two other studies (McLean et al, 2004; Roiser et al, 2005). When an efficiency score was created that reflected a combination of accuracy scores and response times, we found that efficiency of responding to negative relative to positive stimuli was enhanced by ATPD. We had hoped to shed light on the mechanism underlying this relative biasing of attention and response patterns. If positive stimuli are underdetected in ATPD, then individuals in that condition may be experiencing an anhedonic response that, while not apparent in their subjective mood ratings, reflects a decreased ability to appreciate positive environmental cues. On the other hand, if negative stimuli are more vigorously appreciated during ATPD, then the manipulation might be impacting circuits that mediate stress reactivity, leading to general distress. While it would be expedient to explain this phenomenon as a shift in sensitivity from positive to negative affect on a continuous bipolar scale, positive and negative affect have been shown to be unipolar, orthogonal constructs (Tellegen, 1985).

Thus, we were stymied by the fact that while the detection efficiency for positive vs negative stimuli appears to be inverted by the manipulation, neither affective domain in and of itself is significantly impacted as a main effect. Comparisons with a neutral condition yielded no differences between responses to positive $v s$ neutral words, but comparisons did suggest that perhaps responsiveness to negative stimuli is heightened by the manipulation. Again, the magnitude of this effect is underwhelming. This could be due to statistical power issues that could be resolved with much larger samples than are typically used in these types of studies. Alternatively, it may be the case that affective processing for both positive and negative stimuli may be impacted by ATPD in a manner that resembles a depressogenic profile, namely decrements in processing of positive stimuli and facilitation of negative stimuli processing (Harmer et al, 2003; Watson et al, 1988). The lower $d^{\prime}$ scores for positive words for individuals in the ATPD condition, albeit at a trend level, support the proposed association between low DA levels and anhedonic depression, a suggestion that is substantiated by neuroleptics' induction of anhedonia (Depue and Iacono, 1989). On the other hand, the higher hit rate that we observed for negative words, in the context of faster responses to these stimuli, suggests heightened attention to unpleasant environmental stimuli. Although speculative, this profile might also be related to the increases in alertness and hostility in the ATPD group, which could be conceptualized as a form of vigilance (although ratings of alertness and hostility did not correlate significantly with task performance). Given that others have found similar depressive-like dysregulation of affective processing following ATPD (McLean et al, 2004; Roiser et al, 2005), the legitimacy of the finding is strongly supported. This pattern of findings furthers longstanding hypotheses regarding a contributory role for poor dopaminergic tone in the etiology of depression (Depue and Iacono, 1989), and additionally suggests biases in the processing of incoming affective stimuli as a potential mechanism. Additional research using considerably larger samples or meta-analytic strategies to combine data across laboratories may shed light on the means by which ATPD impacts the processing of positive $v s$ negative stimuli and whether dysregulation within one affective domain leads subsequently to dysregulation in the other one.

Our third major finding is that individuals in the ATPD condition demonstrated an effect on cognitive control processes that has not been previously reported. We observed that the ATPD group demonstrated a significantly reduced sensitivity $\left(d^{\prime}\right)$ while performing a Go No-Go task. Specifically, their hit rate was lower than that of individuals in the BAL condition in the No-Go portion of a nonaffective go/no-go task. The No-Go portion of the task involved inhibiting responses to some stimuli, while a separate Target Detection portion (which was unaffected by the manipulation) required maintaining a vigilant stance to respond to infrequently occurring targets. A lower hit rate on the No-Go portion of the task implies that participants are failing to respond appropriately under conditions where the prepotent tendency is, in fact, to respond on $80 \%$ of the task's trials. Typically, one expects false alarm rates to distinguish between groups of interest on this portion, because an increased level of control must be exerted to inhibit responses to infrequently occurring letters that are rapidly presented. The lower hit rate that was observed suggests attentional lapses or deficiencies in cognitive control when responses had to be more tightly modulated. Individuals in the ATPD group also showed increased response bias (c) scores relative to those in the $\mathrm{BAL}$ condition. In combination with their lower $d^{\prime}$ scores and lower hit rates, this finding suggests that individuals in the ATPD group had difficulty distinguishing between signal 
and noise demands on this portion of the task and that they adopted a more conservative criterion for responding, leading to a tendency to omit responses.

In examining error patterns between groups, we discovered group differences in the tendency to omit responses as opposed to the tendency to fail to inhibit responding (ie, errors of commission). Specifically, ATPD subjects' errors of omission were significantly more likely to occur for targets immediately (one after) a nontarget, for which they inhibit behavior no differently than controls.

It is unlikely that this difference is caused by pure motor impairment, since no differences were found on either the Target Detection portion of the task, or on the finger tapping test, which measures motor speed in a context where the overwhelming tendency, once responses have been initiated, is to continue to respond with vigor without the need to discriminate between conditions where a response is or is not appropriate. Trials following response inhibition for $\mathrm{X}$ within the No-Go portion of the task appear to have presented participants with a uniquely difficult set of conditions (re-activation following inhibition), which elicited a subtle impairment.

We conclude that the hit rate disparity represents a deficit in cognitive control of motor initiation under conditions where the decision to respond or not, as well as the actual response execution, had to be quickly modulated. It is notable that we also found that individuals in the ATPD condition generated fewer words on the first trial of the COWAT, which required them to verbally generate words at a rapid and internally controlled pace.

This overall pattern is consistent with an influential theoretical explanation of the role of DA in cognition, and with evidence from the Parkinson's disease (PD) literature. A specific role for DA in cognitive control was proposed a number of years ago by Oades (1985), who suggested that DA modulates the neuronal process of switching from one relevant signal to another to facilitate ongoing behavior. Depleted DA levels would lead to a reduced ability to taskswitch due to an inability to appreciate the relevance of incoming motivational signals, while elevated DA levels would augment the processing of new inputs (signals) to facilitate task-switching. Related to this model, Cools et al (2001, 2003) have described task-switching deficits in individuals with PD. They found that when PD patients must respond rapidly to task switches without the benefit of external cueing, they perform in a less flexible manner than control participants. Moreover, this response pattern is ameliorated with L-Dopa therapy, which is presumed to act upon a circuit involving the dorsal striatum and dorsal regions of the prefrontal cortex (Cools et al, 2001; Hayes et al, 1998; Sohn et al, 2000).

Clearly, ATPD does not lead to impairments that approach the severity of what is observed in PD; however, it may be that the manipulation impacts similar neural circuits. The resulting impairment is one that can only be appreciated using sensitive neurocognitive measures. Thus, our findings suggest that ATPD may lead to a very subtle task switching deficit, particularly when the stopping and subsequent re-initiation of behavior is rapidly required. It is notable that in the ATPD group, slower responses were associated with higher hit rates suggesting that perhaps this group had to exert greater effort in order to integrate their attentional resources and decision criteria with efficient responses. Based on this pattern, additional studies of task switching performance during ATPD are warranted. Moreover, whether this same sort of deficit impacts affective processing in a way that could account for the valence differences described earlier could be addressed by using affective tasks that require faster modulations of responses in the course of making decisions.

Several limitations of our study should be mentioned. First, it would have been useful had we been able to measure amino-acid levels in addition to prolactin in order to demonstrate correlations between changes in the peripheral tyrosine-to-LNAA ratio and prolactin changes. This type of analysis would more strongly support that ATPD is an efficacious method for lowering brain DA levels. Second, our small sample size may have hindered our ability to observe significant correlations between ATPD response (changes in PRL levels) and behavioral or mood changes. This type of demonstration would also be important in validating ATPD. Our correlational analyses suggest that individuals with higher negative emotionality and lower behavioral constraint may be most vulnerable to the effects of ATPD, but these impressions warrant replication given our sample size. Finally, we were conservative in our exclusion of participants with out-of-range predrink prolactin data. We attributed these high premanipulation levels as indicative of anxiety given that we excluded individuals with endocrine problems and hormone use, and we controlled for menstrual cycle variations in female subjects. Perhaps our focus on maximally healthy individuals decreased the variability in affective processing that would otherwise have been observed as a consequence of the manipulation. This conservative strategy can be viewed as both a weakness and a strength, since we may have underestimated the extent of ATPD's effects. On the other hand, our observed findings are even more striking given our stringent level of experimental control.

In summary, we report novel deficits in a behavioral initiation component of a go/no-go task and dysregulated affective processing, but not changes in mood as effects of ATPD. Overall, the pattern of findings reported herein is consistent with findings from PET studies that suggest that ATPD operates most strongly in limbic and striatal circuitry and with models of DA functioning that suggest that it serves to switch relevant signals into information-processing networks when these signals are new and, in the context of ongoing behavior, from node-to-node within information processing circuits that are active. A better understanding of the implications of this pattern of findings is a primary target for additional investigations into how tyrosine depletion impacts dopaminergic functioning within neural circuits that facilitate response modulation and affective control.

\section{ACKNOWLEDGEMENTS}

This work was supported by a McKnight Land-Grant Professorship awarded to M Luciana, by a University of Minnesota Graduate School Fellowship and a National Science Foundation Graduate Research Fellowship to S Vrshek-Schallhorn, and by Grant M01-RR0400 from the 
National Center for Research Resources, National Institute of Health awarded to the General Clinical Research Center at the University of Minnesota. Address correspondence to either Monica Luciana (lucia003@umn.edu) or Suzanne Vrshek-Schallhorn (vrsh0001@umn.edu).

\section{REFERENCES}

Benton AL, Hamsher KD (1976). Multilingual Aphasia Examination. University of Iowa: Iowa City.

Boado RJ, Li JY, Nagaya M, Zhang C, Pardridge WM (1999). Selective expression of the large neutral amino acid transporter at the blood-brain barrier. Proc Natl Acad Sci USA 96: 1207912084.

Bond A, Lader M (1974). The use of analogue scales in rating subjective feelings. Br J Med Psychol 47: 211-218.

Bradley MM, Lang PJ (1999). Affective Norms for English Words $(A N E W)$. The NIMH Center for the Study of Emotion and Attention, University of Florida: Gainesville, FL.

Braver TS, Barch DM, Gray JR, Molfese DL, Snyder A (2001). Anterior cingulate cortex and response conflict: effects of frequency, inhibition and errors. Cerebral Cortex 11: 825-836.

Carver CS, White TL (1994). Behavioral inhibition, behavioral activation, and affective responses to impending reward and punishment: the BIS/BAS scales. J Pers Soc Psychol 67: 319-333.

Cools R, Barker RA, Sahakian BJ, Robbins TW (2001). Enhanced or impaired cognitive function in Parkinson's disease as a function of dopaminergic medication and task demands. Cerebral Cortex 11: 1136-1143.

Cools R, Barker RA, Sahakian BJ, Robbins TW (2003). l-Dopa medication remediates cognitive inflexibility, but increases impulsivity in patients with Parkinson's disease. Neuropsychologia 41: 1431-1441.

Depue RA, Iacono WG (1989). Neurobehavioral aspects of affective disorders. Ann Rev Psychol 40: 457-492.

Ellenbogen MA, Young SN, Dean P, Palmour RM, Benkelfat C (1996). Mood response to acute tryptophan depletion in healthy volunteers: seex differences and temporal stability. Neuropsychopharmacology 15: 465-474.

Fernstrom M, Fernstrom J (1995). Acute tyrosine depletion reduces tyrosine hydroxylation rate in rat central nervous system. Life Sci 57: 97-102.

First MB, Spitzer RL, Gibbon M, Williams JBW (1997). Structured Clinical Interview for DSM-IV Axis I Disorders-Patient Edition (SCID-I/P, Version 2.0, 4.97 Revision). New York State Psychiatric Institute, Biometrics Research Department: New York.

Freeman ME, Kanyicska B, Lerant A, Nagy G (2000). Prolactin: structure, function, and regulation of secretion. Physiol Rev 80: 1523-1631.

Harmer CJ, Hill SA, Taylor MJ, Cowen PJ, Goodwin GM (2003). Toward a neuropsychological theory of antidepressant drug action: increase in positive emotional bias after potentiation of norepinephrine activity. Am J Psychiatry 160: 990-992.

Harmer CJ, McTavish SF, Clark L, Goodwin GM, Cowen PJ (2001). Tyrosine depletion attenuates dopamine function in healthy volunteers. Psychopharmacology 154: 105-111.

Harrison BJ, Olver JS, Norman TR, Burrows GD, Wesnes KA, Nathan PJ (2004). Selective effects of acute serotonin and catecholamine depletion on memory in healthy women. J Psychopharmacol 18: 32-40.

Hayes AE, Davidson MC, Keele SW, Rafal RD (1998). Towards a functional analysis of the basal ganglia. J Cogn Neurosci 10: $178-198$.

Jaskiw GE, Bongiovanni R (2004). Brain tyrosine depletion attenuates haloperidol-induced striatal dopamine release in vivo and augments haloperidol-induced catalepsy in the rat. Psychopharmacology 172: 100-107.
Knutson B, Wolkowitz OM, Cole SW, Chan T, Moore EA, Johnson $\mathrm{RC}$ et al (1998). Selective alteration of personality and social behavior by serotonergic intervention. Am J Psychiatry 155: 373-379.

Le Masurier M, Cowen PJ, Sharp T (2004b). Fos immunocytochemical studies on the neuroanatomical sites of action of acute tyrosine depletion in the rat brain. Psychopharmacology 171: 435-440.

Le Masurier M, Houston G, Cowen PJ, Grasby PM, Sharp T, Hume $S$ (2004a). Tyrosine-free amino acid mixture attenuates amphetamine-induced displacement of [11C]Raclopride in striatum in vivo: a rat PET study. Synapse 51: 151-157.

Leyton M, Dagher A, Boileau I, Casey K, Baker GB, Diksic M et al (2004). Decreasing amphetamine-induced dopamine release by acute phenylalanine/tyrosine depletion: a PET/[11C]raclopride study in healthy men. Neuropsychopharmacology 29: 427-432.

Leyton M, Young SN, Pihl RO, Etezadi S, Lauze C, Blier P et al (1999). Effects on mood of acute phenylalanine/tyrosine depletion in healthy women. Neuropsychopharmacology 22: 52-63.

Lorr M, McNair DM, Fisher S (1982). Evidence for bipolar mood states. J Pers Assess 46: 432-436.

Lorr M, McNair DM, Heuchert JWP (2003). Manual Supplement for the Profile of Mood States: Bipolar Form. Mutli-Health Systems: Toronto.

Luciana M, Collins P (1997). Dopaminergic modulation of working memory for spatial but not object cues in normal humans. J Cogn Neurosci 9: 330-367.

Luciana M, Vrshek-Schallhorn S, Wahlstrom D, White T (2005). Acute tyrosine depletion and dopamine-modulated cognitive functions in healthy volunteers. Paper presented at the Cognitive Neuroscience Society, New York, NY.

Lythe KE, Anderson IM, Deakin JFW, Elliott R, Strickland PL (2005). Lack of behavioral effects after acute tyrosine depletion in healthy volunteers. J Psychopharmacol 19: 5-11.

McLean A, Rubinstein JS, Robbins TW, Sahakian BJ (2004). The effects of tyrosine depletion in normal healthy volunteers: implications for unipolar depression. Psychopharmacology 171: 286-297.

McTavish SF, Callado L, Cowen PJ, Sharp T (1999b). Comparison of the effects of a-methyl- $p$-tyrosine and a tyrosine-free amino acid load on extracellular noradrenaline in the rat hippocampus in vivo. J Psychopharmacol 13: 379-384.

McTavish SF, Cowen PJ, Sharp T (1999a). Effects of a tyrosine-free amino acid mixture on regional brain synthesis and release. Psychopharmacology 141: 182-188.

McTavish SF, Mannie Z, Cowen PJ (2004). Tyrosine depletion does not cause depressive relapse in antidepressant-treated patients. Psychopharmacology 175: 124-126.

McTavish SF, Mannie Z, Harmer CJ, Cowen PJ (2005). Lack of effect of tyrosine depletion on mood in recovered depressed women. Neuropsychopharmacology 30: 786-791.

McTavish SF, McPherson MH, Harmer CJ, Clark L, Sharp T, Goodwin GM et al (2001b). Antidopaminergic effects of dietary tyrosine depletion in healthy subjects and patients with manic illness. Br J Psychiatry 179: 356-360.

McTavish SF, Raumann B, Cowen PJ, Sharp T (2001a). Tyrosine depletion attenuates the behavioural stimulant effects of amphetamine and cocaine in rats. Eur J Pharmacol 424: 112-119.

Mehta MA, Swainson R, Ogilvie AD, Sahakian BJ, Robbins TW (2001). Improved short-term spatial meory but impaired reversal learning following the dopamine D2 agonist bromocriptine in human volunteers. Psychopharmacology 159: 10-20.

Moja EA, Lucini V, Benedetti F, Lucca A (1996). Decrease in plasma phenylalanine and tyrosine after phenylalanine-tyrosine free amino acid solutions in man. Life Sci 58: 5389-5395.

Montgomery AJ, McTavish SF, Cowen PJ, Grasby PM (2003). Reduction of brain dopamine concentration with dietary 
tyrosine plus phenylalanine depletion: an $\left[{ }^{11} \mathrm{C}\right]$ Raclopride PET study. Am J Psychiatry 160: 1887-1889.

Murphy FC, Sahakian BJ, Rubinstein JS, Michael A, Rogers RD, Robbins TW et al (1999). Emotional bias and inhibitory control processes in mania and depression. Psychol Med 29: 1307-1321.

Oades RD (1985). The role of noradrenaline in tuning and dopamine in switching between signals in the CNS. Neurosci Biobehav Rev 9: 261-282.

Palmour RM, Ervin FR, Baker GB, Young SN (1998). An amino acid mixture deficient in phenylalanine and tyrosine reduces cerebrospinal fluid catecholamine metabolites and alcohol consumption in vervet monkeys. Psychopharmacology 136: 1-7.

Patrick CJ, Curtin JJ, Tellegen A (2002). Development and validation of a brief form of the Multidimensional Personality Questionnaire. Psychol Assessment 14: 150-163.

Reitan RM, Davison LA (1974). Clinical Neuropsychology: Current Status and Applications. Hemisphere: New York.

Roiser JP, McLean A, Ogilvie AD, Blackwell AD, Bamber DJ, Goodyer I et al (2005). The subjective and cognitive effects of acute phenylalanine and tyrosine depletion in patients recovered from depression. Neuropsychopharmacology 30: 775-785.

Sheehan BD, Tharyan P, McTavish SF, Campling GM, Cowen PJ (1996). Use of a dietary manipulation to deplete plasma tyrosine and phenylalanine in healthy subjects. J Psychopharmacol 10: 231-234.
Sohn M-H, Ursu S, Anderson JR, Stenger VA, Carter CS (2000). The role of prefrontal cortex and posterior parietal cortex in task switching. Proc Natl Acad Sci USA 97: 13448-13453.

Stanislaw H, Todorov N (1999). Calculation of signal detection theory measures. Behav Res Methods Instr Comput 31: 137-149.

Tellegen A (1985). Structures of mood and personality and their relevance to assessing anxiety, with an emphasis on self-report. In Tuma AH, Maser JD (eds). Anxiety and the Anxiety Disorders. Lawrence Erlbaum Associates: Hillsdale, NJ, pp 681-706.

Torner L, Moloumby R, Nava G, Aranda J, Clapp C, Neumann ID (2004). In vivo release and gene upregulation of brain prolactin in response to physiological stimuli. Eur J Neurosci 19: 16011608.

Watson D, Clark L, Carey G (1988). Positive and negative affectivity and their relation to anxiety and depressive disorders. J Abnormal Psychol 97: 346-353.

Watson D, Clark LA (1994). Manual for the Positive and Negative Affect Schedule-Expanded Form. The University of Iowa: Iowa City.

Watson D, Tellegen A (1985). Towards a consensual structure of mood. Psychol Bull 98: 219-235.

Wechsler D (1997). The Wechsler Adult Intelligence Scale, Third Revision. The Psychological Corporation: San Antonio, TX. 